\title{
The wheat leaf epidermal pattern as a model for studying the effect of stress conditions on morphogenesis
}

\author{
Zubairova U. ${ }^{1,2 *}$, Doroshkov A. ${ }^{1,2}$ \\ ${ }^{1}$ Institute of Cytology and Genetics, SB RAS, Novosibirsk, Russia \\ ${ }^{2}$ Novosibirsk State University, Novosibirsk, Russia \\ *e-mail: ulyanochka@bionet.nsc.ru
}

\begin{abstract}
Motivation and Aim: The leaf epidermis of cereals is a widely used model system for studying the mechanisms of pattern formation for plant tissues, as it contains readily observable specialized cells. In this work we used a growing wheat leaf to study stressinduced dynamic changes in morphogenesis of specialized epidermal cells. In the process of formation, the leaf of wheat remains in the stationary growth phase for long time period. This fact permits us to observe a series of successive morphogenetic events recorded in the cellular structure of the mature leaf.
\end{abstract}

Methods and Algorithms: High-resolution 3D LSM-images allow extracting quantitative characteristics describing the cellular structure of leaf epidermis. However, to obtain a large amount of statistical data methods of high throughput computer based image segmentation should be used. We developed a workflow for detection of structural properties of leaf epidermis from 3D images obtained from confocal laser scanning microscopy. The workflow includes the protocol of sample preparation, image processing ImageJ-plugin [1] and data extraction algorithms.

Results: We showed significant aberrations of stomatal morphogenesis in the epidermis of boot leaves of wheat varieties Saratovskaya 29 and Yanetskis Probat in response to cold stress. We found that nonfunctional stomata predominated in the zone of maximum manifestation of stress, whereas in the zones formed before and after the stress impact, the developmental anomalies come to the disturbance in the morphogenesis of subsidiary cells [2]. In Saratovskaya 29, a significant amount of ectopic trichomes formed in rows predetermined to stoma formation. The proposed approach can provide standardized qualitative and quantitative data on stressinduced morphogenesis aberrations in wheat leaf epidermis. Subsequently, these data can be used for verification of computer models of leaf morphogenesis. Further study of the mechanisms of the effect of cold stress on morphogenesis will add to the search for additional opportunities to increase wheat yields in areas of risky agriculture.

Acknowledgements: The work was supported by the budget project 0324-2019-0040.

\section{References}

1 Zubairova U.S., Verman P.Yu., Oshchepkova P.S., Elsukova A.S., Doroshkov A.V. LSM-W2: Laser Scanning Microscopy Worker for Wheat Leaf Surface Morphology. BMC Systems Biology. 2019; 13(Suppl 1):22. DOI 10.1186/s12918-019-0689-8.

2 Zubairova U.S., Doroshkov A.V. Wheat leaf epidermal pattern as a model for studying the influence of stress conditions on morphogenesis. Vavilovskii Zhurnal Genetiki $i$ Selektsii = Vavilov Journal of Genetics and Breeding. 2018;22(7):837-844. DOI 10.18699/VJ18.32-o (in Russian). 